MATHEMATICS OF COMPUTATION

Volume 67, Number 224, October 1998, Pages 1707-1726

S $0025-5718(98) 01007-2$

\title{
PERTURBING POLYNOMIALS WITH ALL THEIR ROOTS ON THE UNIT CIRCLE
}

MICHAEL J. MOSSINGHOFF, CHRISTOPHER G. PINNER, AND JEFFREY D. VAALER

\begin{abstract}
Given a monic real polynomial with all its roots on the unit circle, we ask to what extent one can perturb its middle coefficient and still have a polynomial with all its roots on the unit circle. We show that the set of possible perturbations forms a closed interval of length at most 4 , with 4 achieved only for polynomials of the form $x^{2 n}+c x^{n}+1$ with $c$ in $[-2,2]$. The problem can also be formulated in terms of perturbing the constant coefficient of a polynomial having all its roots in $[-1,1]$. If we restrict to integer coefficients, then the polynomials in question are products of cyclotomics. We show that in this case there are no perturbations of length 3 that do not arise from a perturbation of length 4 . We also investigate the connection between slightly perturbed products of cyclotomic polynomials and polynomials with small Mahler measure. We describe an algorithm for searching for polynomials with small Mahler measure by perturbing the middle coefficients of products of cyclotomic polynomials. We show that the complexity of this algorithm is $O\left(C^{\sqrt{d}}\right)$, where $d$ is the degree, and we report on the polynomials found by this algorithm through degree 64 .
\end{abstract}

\section{INTRODUCTION}

In this paper we study the effect of slightly perturbing the middle coefficient of a polynomial having all its roots on the unit circle $\mathbf{T}=\{z \in \mathbf{C}:|z|=1\}$. We first consider polynomials with real coefficients. Let $f$ be a monic real polynomial with even degree $2 N$ having all its roots in $\mathbf{T}$, and let

$$
B(f)=\left\{\beta \in \mathbf{C}: \text { all zeros of } f(x)-\beta x^{N} \text { occur in } \mathbf{T}\right\} .
$$

We show (Theorem 2.1) that $B(f)$ is a closed real interval of length at most 4 .

Let $f$ be a polynomial with complex coefficients and degree $d$,

$$
f(x)=a \prod_{n=1}^{d}\left(x-\alpha_{n}\right) .
$$

The Mahler measure of $f$ is

$$
M(f)=|a| \prod_{n=1}^{d} \max \left\{1,\left|\alpha_{n}\right|\right\}=\exp \left(\int_{0}^{1} \log \left|f\left(e^{2 \pi i t}\right)\right| d t\right) .
$$

If $f$ has integer coefficients, then clearly $M(f) \geq 1$, and a well-known theorem of Kronecker (1857) shows that $M(f)=1$ if and only if $f(x)$ is a product of cyclotomic

Received by the editor February 7, 1997.

1991 Mathematics Subject Classification. Primary: 26C10; Secondary: 12-04, 12D10, 30C15.

Key words and phrases. Cyclotomic, Mahler measure, Lehmer's problem, transfinite diameter.

(C) 1998 American Mathematical Society 
polynomials and the monomials $\pm x$. In 1933, D. H. Lehmer [7] asked if there exist polynomials having integer coefficients with Mahler measure arbitrarily close to 1 . No polynomials have been found with measure greater than 1 and smaller than the degree 10 polynomial noted by Lehmer,

$$
M\left(x^{10}+x^{9}-x^{7}-x^{6}-x^{5}-x^{4}-x^{3}+x+1\right)=1.1762808 \ldots
$$

The best result in this direction (up to the constant $c$ ) is the bound

$$
M(f) \geq 1+c\left(\frac{\log \log d}{\log d}\right)^{3}
$$

of Dobrowolski [5].

A polynomial $f$ of degree $d$ is reciprocal if $f(x)=x^{d} f(1 / x)$. Smyth [15] proved that if an irreducible polynomial has Mahler measure greater than 1 and less than $1.3247 \ldots$ (the real root of $x^{3}-x-1$ ), then $f$ is a reciprocal polynomial. This fact, combined with the observation that the Mahler measure is multiplicative, allows us to restrict our attention to monic, irreducible, reciprocal polynomials of even degree when searching for polynomials with small Mahler measure.

Let $\Phi_{n}(x)$ denote the $n$th cyclotomic polynomial

$$
\Phi_{n}(x)=\prod_{\substack{k=1 \\(k, n)=1}}^{n}\left(x-\zeta_{n}^{k}\right), \quad \zeta_{n}=e^{2 \pi i / n} .
$$

It is implicit in the proof of (1.1) by Rausch [13] and explicit in the work of Silverman [14] that if $\alpha$ is a root of a polynomial with small Mahler measure, then not only is $\alpha$ a unit but $\Phi_{m}(\alpha)$ should probably also be a unit for many small $m$ (that is, $\alpha$ should behave very much like a root of unity). Now if $\alpha$ is a root of the perturbed cyclotomic product

$$
\prod_{n \geq 1} \Phi_{n}(x)^{e(n)} \pm x^{N}
$$

where $\sum_{n \geq 1} e(n) \varphi(n)=2 N$, then $\Phi_{m}(\alpha)$ is a unit for all $m$ for which $e(m) \neq 0$ (observe that the resultant of the polynomial with $\Phi_{m}(x)$ is \pm 1 ). Thus we might expect such polynomials to be good candidates for small Mahler measure.

It is worth remarking that the examples

$$
\prod_{m=1}^{M} \Phi_{m}(x) \pm x^{N}, \quad 2 N=\sum_{m=1}^{M} \varphi(m)
$$

straightforwardly show that Silverman's bound $O\left(d^{1+0.7 / \log \log d}\right)$ on the number of $m$ such that $\Phi_{m}(\alpha)$ is a unit where $\alpha$ is not a root of unity cannot be reduced beyond $\pi \sqrt{d / 3}+O(\log d)$ (we are not aware of a better lower bound).

We note that Lehmer's polynomial may be written

$$
\Phi_{1}^{2}(x) \Phi_{2}^{2}(x) \Phi_{3}^{2}(x) \Phi_{6}(x)-x^{5} .
$$

In $\S 3$ we observe that many of the known polynomials with small Mahler measure are in fact slightly perturbed products of cyclotomic polynomials. This suggests a method for searching for new polynomials with small measure. We describe an algorithm for constructing all polynomials of a given degree $d$ that are products of cyclotomic polynomials, subject to certain restrictions on the multiplicity of the factors, then testing the Mahler measure of slight perturbations of these polynomials. 
We show (Theorem 3.1) that the number of polynomials of degree $d$ considered by this algorithm is $O\left(C^{\sqrt{d}}\right)$, and we describe the polynomials found by implementing the algorithm and searching through degree 64 . Although many new polynomials with small Mahler measure are found, none is smaller than Lehmer's degree 10 example.

Finally, we consider products of cyclotomic polynomials whose roots remain on the unit circle when their middle coefficient is changed by an integer amount. We show (Theorem 4.1) that if $f$ allows a shift of length 3, then in fact it allows a shift of length 4 , and we characterize the polynomials that allow a shift this large. We also derive some restrictions on polynomials allowing a shift of length 2 , and we describe the polynomials found by our algorithm that allow a shift of 1 or 2 .

\section{Polynomials With Real COefFicients}

We prove the following theorem.

Theorem 2.1. Let $f$ be a monic polynomial of degree $2 N \geq 2$ with real coefficients and all its roots on the unit circle. The set $B(f)$ is a closed interval in $\mathbf{R}$ of length at most 4 , with 4 achieved only for polynomials of the form $x^{2 N}+c_{1} x^{N}+1$.

In the proof, we first transform our polynomial $f$ with all its roots on the unit circle into a polynomial $p$ with all its roots in the interval $[-1,1]$. Defining

$$
A(p)=\{\alpha \in \mathbf{C}: \text { all zeros of } p(x)-\alpha \text { occur in }[-1,1]\},
$$

we reduce Theorem 2.1 to the following statement.

Theorem 2.2. Let $p$ be a monic polynomial of degree $N \geq 1$ with real coefficients and all its roots in $[-1,1]$. The set $A(p)$ is a closed interval of length at most $2^{2-N}$, with equality only when $p(x)=2^{1-N} T_{N}(x)+c_{2}$ for some constant $c_{2}$, where $T_{N}(x)$ is the N th Chebyshev polynomial.

\subsection{Reduction of Theorem 2.1 to Theorem 2.2. Suppose}

$$
f(z)=\sum_{n=0}^{2 N} a_{n} z^{n}
$$

is a monic polynomial in $\mathbf{R}[z]$ of degree $2 N \geq 2$ having all its roots in $\mathbf{T}$. It follows easily that $f(0)=(-1)^{m}$, where $m$ is the multiplicity with which $f$ has a zero at 1 , and that

$$
z^{2 N} f\left(z^{-1}\right)=f(0) f(z) .
$$

Now if $f(0)=-1$ we must have $a_{n}=-a_{2 N-n}$, for $0 \leq n \leq N$. In this case $a_{N}=0$ and thus $B(f)=\{0\}$. Hence we shall assume $f(0)=1$ and that $f$ is reciprocal. We note that in this case the multiplicities with which $f$ has zeros at 1 and -1 are consequently even.

Writing $z=\exp (2 \pi i \theta)$, we have

$$
\begin{aligned}
z^{-N} f(z) & =a_{N}+2 \sum_{n=1}^{N} a_{N+n} \cos 2 \pi n \theta \\
& =a_{N}+2 \sum_{n=1}^{N} a_{N+n} T_{n}(\cos 2 \pi \theta),
\end{aligned}
$$


where

$$
T_{n}(z)=\sum_{m=0}^{n} t_{n m} z^{m}, \quad t_{n n}=2^{n-1},
$$

is the $n$th Chebyshev polynomial. Thus

$$
\begin{aligned}
z^{-N} f(z) & =a_{N}+2 \sum_{n=1}^{N} a_{N+n} t_{n, 0}+2 \sum_{m=1}^{N}\left(\sum_{n=m}^{N} a_{N+n} t_{n m}\right)(\cos 2 \pi \theta)^{m} \\
& =2^{N} p(\cos 2 \pi \theta),
\end{aligned}
$$

where $p(z)$ is a monic polynomial of degree $N$ with all its zeros in $[-1,1]$. More precisely, if $f(z)$ has a zero at -1 with multiplicity $2 k$, a zero at 1 with multiplicity $2 l$, and distinct zeros in the upper half plane at $e^{2 \pi i \alpha_{1}}, e^{2 \pi i \alpha_{2}}, \ldots, e^{2 \pi i \alpha_{M}}$, with multiplicities $\mu_{1}, \mu_{2}, \ldots, \mu_{M}$, respectively, then $p(z)$ has a zero at -1 with multiplicity $k$, a zero at 1 with multiplicity $l$ and zeros at $\cos \left(2 \pi \alpha_{m}\right)$ with multiplicity $\mu_{m}$, for each $m$.

Thus

$$
\begin{aligned}
B(f) & =\left\{\beta \in \mathbf{C}: \text { all zeros of } z^{-N} f(z)-\beta \text { occur in } \mathbf{T}\right\} \\
& =\left\{\alpha \in \mathbf{C}: \text { all zeros of } p(z)-2^{-N} \alpha \text { occur in }[-1,1]\right\} \\
& =2^{N} A(p) .
\end{aligned}
$$

Clearly, the polynomial $f(x)=x^{2 N}+c x^{N}+1$ corresponds to the polynomial $p(x)=2^{-N}\left(2 T_{N}(x)+c\right)$ under this pairing. Therefore Theorem 2.1 follows from Theorem 2.2.

\subsection{Proof of Theorem 2.2.}

Lemma 2.3. Suppose that $p(z)$ has a zero of multiplicity greater than or equal to 3 . Then $A(p)=\{0\}$.

Proof. This is immediate from [11, Problem 49, Part V, Chap. 1] on expanding $p(z)$ about the point in question.

Lemma 2.4. Suppose that $p(z)$ has a zero of multiplicity 2 at either 1 or -1 . Then $A(p)=\{0\}$.

Proof. Suppose that $p(z)-\alpha$ has zeros only in $[-1,1]$, and $p^{\prime}(1)=0$. By Lucas's Theorem, the zeros of $p^{\prime}(z)$ lie in the convex hull of the zeros of $p(z)-\alpha$, so $p(z)-\alpha$ continues to have a zero at 1 . Hence $p(1)-\alpha=-\alpha=0$.

In view of these lemmas we need only prove Theorem 2.2 under the additional assumptions that $p$ has zeros of multiplicity at most 2 and that the zeros at -1 and 1 are of multiplicity 0 or 1 .

It follows that $p^{\prime}(z)$ has only simple zeros: if $p$ has $k$ distinct roots and $N-k$ double roots then, by Rolle's Theorem, $p^{\prime}$ has at least $k-1$ distinct roots between the roots of $p$ in addition to $N-k$ roots at the double roots of $p$; hence each of these $N-1$ must have multiplicity one. Moreover these roots all lie in $(-1,1)$.

Now let $-1 \leq \xi_{1}<\xi_{2}<\cdots<\xi_{M} \leq 1$ be the distinct zeros of $p$, and let $I_{0}, \ldots$, $I_{M}$ denote the intervals

$$
\left[-1, \xi_{1}\right),\left(\xi_{1}, \xi_{2}\right), \ldots,\left(\xi_{M}, 1\right]
$$


Define $z_{m}(\alpha), m=0, \ldots, M$, to be the number of real zeros, counted with multiplicity, of $p(z)-\alpha$ in $I_{m}$. The number of real zeros of $p(z)-\alpha, \alpha \neq 0$, in $[-1,1]$ is then

$$
\sum_{m=0}^{M} z_{m}(\alpha)
$$

Since each interval $I_{m}$ contains at most one root of $p^{\prime}(z)$, the functions $z_{m}(\alpha)$ are integer-valued, nonnegative, and decrease for $\alpha>0$. Thus

$$
\alpha \rightarrow \sum_{m=0}^{M} z_{m}(\alpha)
$$

decreases on $(0, \infty)$. A similar argument holds for

$$
\alpha \rightarrow \sum_{m=0}^{M} z_{m}(-\alpha)
$$

Thus $A(p)$ is an interval, possibly degenerating to a point.

By the open mapping theorem, $p\{\mathbf{C} \backslash[-1,1]\}$ is open, and $p\{\mathbf{C} \backslash[-1,1]\}=$ $\mathbf{C} \backslash A(p)$, so $A(p)$ is closed.

Finally, we prove that the length of $A(p)$ is at most $2^{2-N}$. Let $E \subseteq \mathbf{C}$ be compact and for each integer $L \geq 1$ define

$$
\delta_{L}(E)=\left\{\sup _{\mathbf{z} \in E^{L}} \prod_{1 \leq k<l \leq L}\left|z_{k}-z_{l}\right|\right\}^{2 / L(L-1)} .
$$

It can be shown that $\left\{\delta_{L}(E)\right\}_{L=1}^{\infty}$ is decreasing, and therefore

$$
\rho(E)=\lim _{L \rightarrow \infty} \delta_{L}(E)
$$

exists. Here $\rho(E)$ is called the transfinite diameter of $E$. Among its most important properties are:

(i) If $E_{1} \subseteq E_{2} \subseteq \mathbf{C}$ are compact, then $\rho\left(E_{1}\right) \leq \rho\left(E_{2}\right)$.

(ii) If $[\alpha, \beta] \subseteq \mathbf{R}$ is a closed interval, then $\rho([\alpha, \beta])=(\beta-\alpha) / 4$.

(iii) If $p(z)=z^{L}+b_{L-1} z^{L-1}+\cdots+b_{0}$ is a monic polynomial in $\mathbf{C}[z], E \subseteq \mathbf{C}$ is compact, and

$$
D=\{\omega \in \mathbf{C}: p(\omega) \in E\},
$$

then $D \subseteq \mathbf{C}$ is compact and

$$
\rho(D)^{L}=\rho(E) .
$$

(iv) Let $\mathcal{P}_{n}$ be the class of monic polynomials in $\mathbf{C}[z]$ with degree $n$. For a subset $E \subseteq \mathbf{C}$ define

$$
M_{n}(E)=\inf _{p \in \mathcal{P}_{n}}\|p(z)\|_{E},
$$

where

$$
\|p(z)\|_{E}=\sup _{z \in E}|p(z)|
$$

Then

$$
\rho(E)=\lim _{n \rightarrow \infty} M_{n}(E)^{1 / n}
$$


These and other properties of $\rho$ are considered in [6, pp. 264-274].

With $p(z)$ as before, let

$$
D(p)=\{\omega \in \mathbf{C}: p(\omega) \in A(p)\} .
$$

Obviously $D(p) \subseteq[-1,1]$. Therefore,

$$
\begin{aligned}
\frac{1}{4} \operatorname{length}(A(p)) & =\rho(A(p))=\rho(D(p))^{N} \\
& \leq \rho([-1,1])^{N}=2^{-N},
\end{aligned}
$$

so the length of $A(p)$ is at most $2^{2-N}$.

Notice that we can only have length $2^{2-N}$ if we have equality in this last inequality and

$$
\rho(D)=\rho([-1,1])=1 / 2 .
$$

We show that this implies that $D(p)=[-1,1]$.

If $D(p) \neq[-1,1]$ then, since $D(p)$ is plainly a finite union of closed subintervals of $[-1,1]$, there must exist an interval $U=[a-2 \delta, a+2 \delta] \subset[-1,1]$ such that

$$
D(p) \subseteq E=[-1,1] \backslash U .
$$

We prove that $\rho(E)<1 / 2$. Define $\mathcal{S}_{n}$ to be the set of roots of the $n$th Chebyshev polynomial $T_{n}(z)$ that lie in the subinterval $V=[a-\delta, a+\delta]$. It is readily seen that $\left|\mathcal{S}_{n}\right|>\lambda n$ for some constant $\lambda(V)>0$ and all sufficiently large $n$.

Recalling that

$$
\left\|T_{n}(z)\right\|_{[-1,1]}=1
$$

we take

$$
q_{2 n}(z)=\left(2^{1-n} T_{n}(z)\right)^{2} \prod_{\alpha \in \mathcal{S}_{n}} \frac{(z-\alpha)^{2}-\delta^{2}}{(z-\alpha)^{2}}
$$

and observe that

$$
\begin{aligned}
\left\|q_{2 n}(z)\right\|_{E} & \leq 2^{2-2 n} \prod_{\alpha \in \mathcal{S}_{n}}\left\|1-\frac{\delta^{2}}{(z-\alpha)^{2}}\right\|_{E} \\
& \leq 2^{2-2 n}\left(1-\frac{\delta^{2}}{4}\right)^{\lambda n} .
\end{aligned}
$$

Hence

$$
\rho(D) \leq \rho(E)=\lim _{n \rightarrow \infty} M_{2 n}(E)^{1 / 2 n} \leq \frac{1}{2}\left(1-\frac{\delta^{2}}{4}\right)^{\lambda / 2}<\frac{1}{2} .
$$

Thus $D(p)=[-1,1]$ and $p([-1,1])=A(p)$. In particular, writing

$$
A(p)=\left[c-2^{1-N}, c+2^{1-N}\right]
$$

we have

$$
\|p(z)-c\|_{[-1,1]}=2^{1-N},
$$

and $2^{1-N} T_{N}(z)$ is the only monic polynomial of degree $N$ with such a small supremum norm on $[-1,1]$. 


\section{Perturbed Cyclotomics with small Mahler measure}

Many of the known polynomials with small Mahler measure are slightly perturbed products of cyclotomic polynomials. Examining the list of 437 polynomials with Mahler measure less than 1.3 and degree at most 32 compiled by Boyd [2], we find that 222 may be written in the form

$$
\prod_{n \geq 1} \Phi_{n}(x)^{e(n)} \pm x^{N}, \quad \sum_{n \geq 1} e(n) \varphi(n)=2 N,
$$

where all but finitely many of the $e(n)$ are 0 . All but 9 of these polynomials have $e(n) \leq 2$ for all $n$ : seven of the exceptional polynomials have $e(2)=4$, the other two have a quadratic cyclotomic with multiplicity 3 .

The Mahler measure of a polynomial in two variables is defined by

$$
\log M(f(x, y))=\int_{0}^{1} \int_{0}^{1} \log \left|f\left(e^{2 \pi i s}, e^{2 \pi i t}\right)\right| d s d t .
$$

Boyd [3] shows that the Mahler measure of a polynomial in two variables is the limit of measures of associated polynomials in one variable:

$$
M(f(x, y))=\lim _{n \rightarrow \infty} M\left(f\left(x, x^{n}\right)\right) .
$$

The one-variable polynomials associated with the three small two-variable measures

$$
\begin{aligned}
M((y z+1)(y+1)(z+1)-y z) & =1.255433 \ldots, \\
M((y z+1)(y+z)+y z) & =1.285734 \ldots, \\
M\left(\left(y^{2}+y+1\right)(y z+1)(y+z)-y^{2} z\right) & =1.315692 \ldots
\end{aligned}
$$

(substituting $y= \pm x^{m}$ and $z= \pm x^{n}$ ) are also slightly perturbed products of cyclotomic polynomials.

These facts suggest searching for polynomials with small Mahler measure by forming all polynomials of the form (3.1) of a given degree $2 N$, with $e(1) \leq 4$, $e(2) \leq 4$, and $e(n) \leq 2$ for $n>2$. Since we may restrict to reciprocal polynomials with even degree, we further require $e(1)$ and $e(2)$ to be even.

3.1. Description of the Algorithm. We are given an even integer $d=2 N$. Define

$$
S_{1}=\left\{(x-1)^{2},(x+1)^{2}, x^{2}+x+1, x^{2}+1, x^{2}-x+1\right\},
$$

and for $2 \leq n \leq N$, let

$$
S_{n}=\left\{\Phi_{r}(x): \varphi(r)=2 n\right\} .
$$

For each $n$, we construct the sets $T_{m, n}$ for $1 \leq m \leq 2\left|S_{n}\right|$ :

$$
T_{m, n}=\left\{\prod_{\Phi_{k} \in S_{n}} \Phi_{k}(x)^{e(k)}: \sum_{\Phi_{k} \in S_{n}} e(k)=m, 0 \leq e(k) \leq 2\right\} .
$$

So for $n \geq 2, T_{m, n}$ is the collection of products of $m$ irreducible cyclotomic polynomials, each of which has degree $2 n$, where no factor appears more than twice. The sets $T_{m, 1}$ are similar, but the linear cyclotomic factors may appear with multiplicity 0,2 , or 4 . The sets $T_{m, n}$ are used repeatedly by the algorithm and are constructed during initialization.

For larger degrees, we further restrict $e(1)$ and $e(2)$ to be at most 2 . This requires removing from the $T_{m, 1}$ those polynomials having $e(1)=4$ or $e(2)=4$. 
We then enumerate all partitions $\vec{m}=\left(m_{1}, \ldots, m_{N}\right)$ of $N$,

$$
\sum_{n=1}^{N} m_{n} n=N
$$

where $0 \leq m_{n} \leq 2\left|S_{n}\right|$ for $1 \leq n \leq N$. For each such partition, we construct all polynomials of the form

$$
f(x)=\prod_{n=1}^{N} f_{n}(x),
$$

where $f_{n}(x) \in T_{m_{n}, n}$, and for each of these polynomials, we test the Mahler measure of

$$
f(x) \pm x^{N} .
$$

We also consider adjustments to the coefficients next to the middle one, forming

$$
\begin{aligned}
& f(x) \pm x^{N-1}\left(x^{2}+1\right), \\
& f(x) \pm x^{N-1}\left(x^{2}+x+1\right), \\
& f(x) \pm x^{N-1}\left(x^{2}-x+1\right) .
\end{aligned}
$$

Nontrivial upper bounds on the Mahler measure of such perturbed products of cyclotomic polynomials can be deduced directly from the work of Amoroso [1].

As in [2], we use the Graeffe root-squaring algorithm to detect most of the polynomials with large Mahler measure quickly. Given a monic polynomial $g$, let $g_{m}$ denote the monic polynomial whose roots are the $2^{m}$ th powers of the roots of $g$. Let $a_{m}(x)$ and $b_{m}(x)$ be polynomials satisfying

$$
g_{m}(x)=a_{m}\left(x^{2}\right)+x b_{m}\left(x^{2}\right) .
$$

Then we compute $g_{m+1}$ using

$$
g_{m+1}(x)=a_{m}^{2}(x)-x b_{m}^{2}(x) .
$$

Clearly, $M\left(g_{m}\right)=M(g)^{2^{m}}$. If $g$ is a monic polynomial of degree $d, g(0)=1$, and $M(g) \leq M$ for a given number $M$, then we see from [2] that

$$
\left|a_{n, m}\right| \leq\left(\begin{array}{l}
d \\
n
\end{array}\right)+\left(\begin{array}{l}
d-2 \\
n-1
\end{array}\right)\left(M^{2^{m}}+M^{-2^{m}}-2\right)
$$

where $a_{n, m}$ is the coefficient of $x^{n}$ in $g_{m}(x)$. Also, if in addition $g$ is reciprocal, $a_{1, m} \geq d-4$, and $m \geq 1$, then

$$
\begin{aligned}
\left|a_{n, m}\right| \leq & \left(\begin{array}{l}
d \\
n
\end{array}\right)+\left(\begin{array}{l}
d-4 \\
n-2
\end{array}\right)\left(M^{2^{m}}+M^{-2^{m}}-2\right) \\
& +2\left(M^{2^{m-1}}+M^{-2^{m-1}}-2\right)\left(\left(\begin{array}{l}
d-4 \\
n-3
\end{array}\right)+\left(\begin{array}{l}
d-4 \\
n-1
\end{array}\right)\right) .
\end{aligned}
$$

In [2], it is required that the $g_{m}$ have no negative real roots in order to assert (3.10), but the proof only requires that any negative real roots appear with multiplicity greater than 1 . This is guaranteed by having $m \geq 1$.

We remark that the Graeffe algorithm also allows us to detect cyclotomic polynomials quickly, since $g_{m+1}=g_{m}$ for sufficiently large $m$ in this case.

For each perturbed cyclotomic product $g$ constructed, we compute up to 10 of the $g_{m}$. If the coefficients of $g_{m}$ fail to satisfy (3.9) or (3.10) with $M=1.3$, we exclude 
$g$ from further consideration. If $g$ survives the 10 iterations of root-squaring, we remove any cyclotomic factors from $g$, then compute its measure if this polynomial has not been found previously. Bairstow's method [16] is first used to estimate the roots of $g$ using machine-precision arithmetic. If the resulting estimate of the measure of $g$ is sufficiently small, we use PARI or Maple to compute $M(g)$ to greater accuracy.

Additional details on the implementation of this algorithm may be found in [9] or $[10]$.

3.2. Analysis of the Algorithm. We prove the following.

Theorem 3.1. The complexity of the algorithm described above given the input d is $O\left(\exp \left(\frac{1+\epsilon}{\pi} \sqrt{70 \zeta(3) d}\right)\right)$ for every $\epsilon>0$ and $\Omega\left(d^{5 / 4} \exp \left(\frac{1}{\pi} \sqrt{70 \zeta(3) d}\right)\right)$.

In order to prove this theorem, we show

Theorem 3.2. Let $r_{2}(n)$ denote the number of reciprocal polynomials of degree $2 n$ that are products of cyclotomic polynomials, where no cyclotomic factor appears with multiplicity greater than 2 . Then

$$
r_{2}(n) \sim \frac{2}{9 \sqrt{3} \pi}(35 \zeta(3))^{1 / 4} n^{-3 / 4} \exp \left(\frac{2}{\pi} \sqrt{35 \zeta(3) n}\right) .
$$

Substituting $d=2 n$ yields an asymptotic estimate for the number of polynomials of degree $d$ constructed by the algorithm:

$$
\frac{4}{9 \sqrt{3} \pi}\left(\frac{35}{2} \zeta(3)\right)^{1 / 4} d^{-3 / 4} \exp \left(\frac{1}{\pi} \sqrt{70 \zeta(3) d}\right) .
$$

The Graeffe algorithm has complexity $\Theta\left(d^{2}\right)$ (that is, both $O\left(d^{2}\right)$ and $\Omega\left(d^{2}\right)$ ), and the remaining parts of the algorithm have complexity that is polynomial in $d$, so Theorem 3.1 follows from Theorem 3.2.

Let $c(n)$ denote the number of polynomials of degree $n$ that are products of cyclotomic polynomials, and let $P(z)$ be the generating function for these numbers,

$$
P(z)=\sum_{n \geq 0} c(n) e^{-n z},
$$

taking $c(0)=1$. Boyd and Montgomery [4] show that as $z$ tends to 0 in

$$
\mathcal{R}=\left\{z \in \mathbf{C}: \operatorname{Re} z>0,|\arg z| \leq \frac{\pi}{2}-\frac{C}{\log \log (1 /|z|)}\right\}
$$

where $C$ is a fixed positive constant, then

$$
P(z)=a\left(\frac{z}{\log (1 / z)}\right)^{1 / 2} \exp (b / z)\left(1+O\left(\frac{\log \log (1 /|z|)}{\log (1 /|z|)}\right)\right),
$$

where $a=\left(2 \pi e^{\gamma}\right)^{-1 / 2}, b=\zeta(2)^{2} \zeta(3) / \zeta(6)$, and $\gamma$ is Euler's constant. They use this to derive an asymptotic formula for $c(n)$, finding

$$
c(n)=\frac{A \exp (B \sqrt{n})}{n \sqrt{\log n}}\left(1+O\left(\frac{\log \log n}{\log n}\right)\right),
$$

as $n \rightarrow \infty$, where

$$
A=\frac{1}{4 \pi^{2}}\left(\frac{105 \zeta(3)}{e^{\gamma}}\right)^{1 / 2} \quad \text { and } \quad B=\frac{1}{\pi}(105 \zeta(3))^{1 / 2} .
$$


Let $r(n)$ denote the number of reciprocal polynomials of degree $2 n$ that are products of cyclotomic polynomials, and define

$$
R(z)=\sum_{n \geq 0} r(n) e^{-n z}
$$

and

$$
R_{2}(z)=\sum_{n \geq 0} r_{2}(n) e^{-n z}
$$

taking $r(0)=r_{2}(0)=1$.

Lemma 3.3. As $z$ approaches 0 in $\mathcal{R}$,

$$
\begin{aligned}
R(z) & \sim \frac{a}{4 \sqrt{2}}\left(\frac{z}{\log (1 / z)}\right)^{1 / 2} \exp (2 b / z) \\
R_{2}(z) & \sim \frac{4}{9 \sqrt{3}} \exp (4 b / 3 z)
\end{aligned}
$$

where $a=\left(2 \pi e^{\gamma}\right)^{-1 / 2}$ and $b=\zeta(2)^{2} \zeta(3) / \zeta(6)$.

Proof. In product form,

$$
\begin{aligned}
R(z) & =\left(\sum_{l \geq 0} e^{-l z}\right)^{2} \prod_{m \geq 3}\left(\sum_{l \geq 0} \exp (-l \varphi(m) z / 2)\right) \\
& =(1-\exp (-z))^{-2} \prod_{m \geq 3}(1-\exp (-\varphi(m) z / 2))^{-1} .
\end{aligned}
$$

Similarly,

$$
P(z)=\prod_{m \geq 1}(1-\exp (-\varphi(m) z))^{-1}
$$

Thus $R(z)=(1+\exp (-z / 2))^{-2} P(z / 2)$, and (3.13) follows from (3.12).

Now

$$
\begin{aligned}
\frac{R(z)}{R(3 z)} & =\left(1+e^{-z}+e^{-2 z}\right)^{2} \prod_{m \geq 3}(1+\exp (-\varphi(m) z / 2)+\exp (-\varphi(m) z)) \\
& =\left(\frac{1+e^{-z}+e^{-2 z}}{1+e^{-z}}\right)^{2} R_{2}(z)
\end{aligned}
$$

and

$$
\frac{R(z)}{R(3 z)} \sim \frac{1}{\sqrt{3}} \exp (4 b / 3 z)
$$

so (3.14) follows.

Lemma 3.4. $r_{2}(n)$ is increasing. 
Proof. We have

$$
\begin{aligned}
R_{2}(z)=\left(1+e^{-z}\right)^{2} \prod_{m \geq 3}(1+\exp (-\varphi(m) z / 2)+\exp (-\varphi(m) z)) \\
=\left(1+e^{-z}\right)^{2}\left(\prod_{l \geq 0}\left(1+\exp \left(-3^{l} z\right)+\exp \left(-2 \cdot 3^{l} z\right)\right)\right)^{2} \\
\cdot\left(\prod_{\substack{m \geq 4 \\
(m, 3)=1}}(1+\exp (-\varphi(m) z / 2)+\exp (-\varphi(m) z))\right. \\
\left.\cdot \prod_{l \geq 0}\left(1+\exp \left(-3^{l} \varphi(m) z\right)+\exp \left(-2 \cdot 3^{l} \varphi(m) z\right)\right)\right) \\
=\left(\frac{1+e^{-z}}{1-e^{-z}}\right)^{2} \prod_{\substack{m \geq 4 \\
(m, 3)=1}} \frac{1+\exp (-\varphi(m) z / 2)+\exp (-\varphi(m) z)}{1-\exp (-\varphi(m) z)},
\end{aligned}
$$

where the last step follows from the identity

$$
\prod_{l \geq 0}\left(1+w^{3^{l}}+\left(w^{2}\right)^{3^{l}}\right)=\frac{1}{1-w} .
$$

Thus

$$
\left(1-e^{-z}\right) R_{2}(z)=1+\sum_{n \geq 1}\left(r_{2}(n)-r_{2}(n-1)\right) e^{-n z}
$$

is a generating function for a sequence of positive integers.

Proof of Theorem 3.2. We appeal to Ingham's Tauberian Theorem [12]: suppose $f(z)=\int_{0}^{\infty} e^{-u z} d A(u), A(u)$ is nondecreasing, and

$$
f(z) \sim C(M / z)^{m \beta-1 / 2} \exp \left(\frac{(M / z)^{\beta}}{\beta}\right)
$$

uniformly as $z \rightarrow 0$ in any angle of the form $|y|<r x$, where $z=x+i y, m$ is a real constant, and $r, C, M$, and $\beta$ are positive constants. Then, as $u \rightarrow \infty$,

$$
A(u) \sim C \sqrt{\frac{1-\alpha}{2 \pi}}(u M)^{m \alpha-1 / 2} \exp \left(\frac{(u M)^{\alpha}}{\alpha}\right),
$$

where $\alpha=\beta /(\beta+1)$.

Let

$$
f(z)=\left(1-e^{-z}\right) R_{2}(z)-1=\int_{0}^{\infty} e^{-u z} d r_{2}(\lfloor u\rfloor) .
$$

Since $r_{2}(n)$ is increasing and

$$
f(z) \sim \frac{4 z}{9 \sqrt{3}} \exp \left(\frac{4 b}{3 z}\right)
$$

as $z \rightarrow 0$ in $\mathcal{R}$, we apply Ingham's Theorem with $\beta=1, M=4 b / 3, m=-1 / 2$, and $C=16 b / 27 \sqrt{3}$. We conclude that

$$
r_{2}(n) \sim \frac{C}{\sqrt{4 \pi}}(n M)^{-3 / 4} \exp \left(2(n M)^{1 / 2}\right) .
$$


Substituting for $C$ and $M$ and using $\zeta(2)=\pi^{2} / 6$ and $\zeta(6)=\pi^{6} / 945$ yields (3.11).

Let $s_{2}(n)$ be the number of reciprocal polynomials of degree $2 n$ that are products of cyclotomic polynomials, where no cyclotomic factor appears with multiplicity greater than 2, except possibly the linear cyclotomics, which may have multiplicity as great as 4 . We may analyze $s_{2}(n)$ in the same way (its generating function is $R(z) / R(3 z))$, to find that

$$
s_{2}(n) \sim \frac{1}{2 \sqrt{3} \pi}(35 \zeta(3))^{1 / 4} n^{-3 / 4} \exp \left(\frac{2}{\pi} \sqrt{35 \zeta(3) n}\right) .
$$

Comparison with an Exact Formula. In view of the slowly decaying error term in (3.12), we might expect $r_{2}$ and $s_{2}$ to approach their asymptotic values rather slowly. An exact formula for $s_{2}(n)$ is

$$
s_{2}(n)=\sum_{\vec{m}} \prod_{k=1}^{n}\left|T_{m_{k}, k}\right|,
$$

where the sum is over all partitions $\vec{m}=\left(m_{1}, \ldots, m_{n}\right)$ of $n$. Now $\left|T_{m, k}\right|$ is the number of ways to select $m$ objects from a collection of $\left|S_{k}\right|$ objects, where each object may be selected at most twice. Thus $\left|T_{m, k}\right|$ is the coefficient of $x^{m}$ in $\left(1+x+x^{2}\right)^{\left|S_{k}\right|}$. Writing

$$
\left(1+x+x^{2}\right)^{n}=\sum_{k=0}^{2 n} c_{n, k} x^{k},
$$

we have

$$
\begin{aligned}
c_{n, k} & =c_{n, 2 n-k}, \\
c_{n, k} & =\sum_{i=0}^{k / 2}\left(\begin{array}{c}
n \\
k-i
\end{array}\right)\left(\begin{array}{c}
k-i \\
i
\end{array}\right) .
\end{aligned}
$$

Using these facts, we compute $s_{2}(1500)$ :

2385753783987905281502413981937925622349509368675889581151794867226.

The asymptotic estimate from (3.16) is $2.771 \cdot 10^{66}$, about $16.2 \%$ larger than the actual value. When $n=2000$, our estimate is about $15.1 \%$ larger than the exact number.

3.3. Results of the Search. We have implemented this algorithm in $\mathrm{C}++$ and run it on Sun SPARC and Intel Pentium computers. For degree $d \leq 50$, we allow the linear cyclotomics to have multiplicity 4; for $d>50$ all multiplicities are at most 2. The perturbations (3.6), (3.7), and (3.8) are checked through $d=56$, and the middle coefficient adjustment (3.5) is checked through $d=64$. Our program tested about 700 million polynomials and required approximately 10 weeks of CPU time. We summarize our results.

Our program finds 384 of the 437 polynomials in Boyd's list with Mahler measure less than 1.3 and degree at most 32 , about $88 \%$. We find 1380 polynomials with measure less than 1.3 through degree 64 , about $81 \%$ of the 1714 such polynomials now known [10]. 
PERTURBING POLYNOMIALS WITH ALL THEIR ROOTS ON THE UNIT CIRCLE 1719

TABle 1. Smallest Measures as Perturbed Products of Cyclotomic Polynomials

$$
\begin{aligned}
& \text { Measure Polynomial } \\
& 1.176280 \ldots \quad \Phi_{1}^{2}(x) \Phi_{2}^{2}(x) \Phi_{3}^{2}(x) \Phi_{6}(x)-x^{5} \\
& 1.188368 \ldots \quad \Phi_{1}^{2}(x) \Phi_{2}^{2}(x) \Phi_{3}^{2}(x) \Phi_{4}(x) \Phi_{6}(x) \Phi_{9}(x)+x^{9} \\
& 1.200026 \ldots \quad \Phi_{1}^{2}(x) \Phi_{2}^{2}(x) \Phi_{4}(x) \Phi_{6}(x) \Phi_{7}(x)+x^{7} \\
& 1.201396 \ldots \quad\left(\Phi_{1}^{2}(x) \Phi_{5}^{2}(x) \Phi_{7}(x) \Phi_{10}(x)+x^{10}\right) / \Phi_{6}(x) \\
& 1.202616 \ldots \quad \Phi_{1}^{2}(x) \Phi_{2}^{2}(x) \Phi_{3}(x) \Phi_{4}(x) \Phi_{6}(x) \Phi_{12}(x)+x^{7} \\
& 1.205019 \ldots \quad\left(\Phi_{2}^{2}(x) \Phi_{10}(x) \Phi_{16}(x) \Phi_{26}(x)-x^{13}\right) / \Phi_{12}(x) \\
& 1.207950 \ldots \quad \Phi_{1}^{2}(x) \Phi_{2}^{2}(x) \Phi_{3}(x) \Phi_{4}(x) \Phi_{6}(x) \Phi_{7}(x) \Phi_{9}(x) \Phi_{18}(x)+x^{14} \\
& 1.212824 \ldots \quad\left(\Phi_{1}^{2}(x) \Phi_{3}(x) \Phi_{8}(x) \Phi_{9}(x) \Phi_{13}(x)+x^{13}\right) / \Phi_{14}(x) \\
& 1.214995 \ldots \quad \Phi_{1}^{2}(x) \Phi_{2}^{2}(x) \Phi_{3}(x) \Phi_{5}^{2}(x) \Phi_{6}(x) \Phi_{10}(x)+x^{10} \\
& 1.216391 \ldots \quad \Phi_{1}^{2}(x) \Phi_{2}^{2}(x) \Phi_{3}(x) \Phi_{4}(x) \Phi_{6}(x)+x^{5} \\
& 1.218396 \ldots \quad\left(\Phi_{1}^{2}(x) \Phi_{3}^{2}(x) \Phi_{4}(x) \Phi_{6}(x) \Phi_{7}(x) \Phi_{12}^{2}(x)+x^{12}\right) / \Phi_{10}(x) \\
& 1.218855 \ldots \quad \Phi_{1}^{2}(x) \Phi_{2}^{2}(x) \Phi_{3}(x) \Phi_{4}(x) \Phi_{6}(x) \Phi_{7}(x) \Phi_{10}(x) \Phi_{12}(x)+x^{12} \\
& 1.219057 \ldots \quad\left(\Phi_{1}^{2}(x) \Phi_{3}(x) \Phi_{4}(x) \Phi_{5}(x) \Phi_{44}(x)+x^{15}\right) / \Phi_{14}(x) \\
& 1.219446 \ldots \quad \Phi_{1}^{2}(x) \Phi_{2}^{2}(x) \Phi_{3}^{2}(x) \Phi_{4}^{2}(x) \Phi_{6}(x) \Phi_{12}(x)+x^{9} \\
& 1.219720 \ldots \quad \Phi_{1}^{2}(x) \Phi_{2}^{2}(x) \Phi_{3}(x) \Phi_{4}(x) \Phi_{8}(x) \Phi_{9}(x)+x^{9} \\
& 1.220287 \ldots \quad\left(\Phi_{1}^{2}(x) \Phi_{2}^{2}(x) \Phi_{7}(x) \Phi_{10}(x) \Phi_{14}(x) \Phi_{19}(x)+x^{19}\right) / \Phi_{12}(x) \\
& 1.223447 \ldots \quad \Phi_{1}^{2}(x) \Phi_{2}^{2}(x) \Phi_{3}(x) \Phi_{4}(x) \Phi_{5}(x) \Phi_{6}(x) \Phi_{10}(x) \Phi_{20}(x) \Phi_{42}(x)-x^{19} \\
& 1.223777 \ldots \quad\left(\Phi_{1}^{2}(x) \Phi_{2}^{2}(x) \Phi_{4}(x) \Phi_{6}(x) \Phi_{8}(x) \Phi_{17}(x) \Phi_{18}(x)+x^{17}\right) / \Phi_{15}(x) \\
& 1.224278 \ldots \quad \Phi_{2}^{2}(x) \Phi_{6}(x) \Phi_{18}^{2}(x)+x^{8} \\
& 1.225503 \ldots \quad\left(\Phi_{5}(x) \Phi_{48}(x)+x^{10}\right) / \Phi_{4}(x) \\
& 1.225619 \ldots \quad \Phi_{2}^{2}(x) \Phi_{4}(x) \Phi_{6}(x) \Phi_{10}(x) \Phi_{26}(x) \Phi_{30}(x)-x^{15} \\
& 1.225810 \ldots \quad\left(\Phi_{1}^{2}(x) \Phi_{2}^{2}(x) \Phi_{5}(x) \Phi_{10}(x) \Phi_{14}(x) \Phi_{17}(x)+x^{17}\right) / \Phi_{12}(x) \\
& 1.226092 \ldots \quad \Phi_{2}^{2}(x) \Phi_{4}(x) \Phi_{6}(x) \Phi_{20}(x) \Phi_{26}(x)-x^{13} \\
& 1.226493 \ldots \quad \Phi_{1}^{2}(x) \Phi_{2}^{2}(x) \Phi_{3}(x) \Phi_{6}(x) \Phi_{9}(x) \Phi_{17}(x) \Phi_{18}(x)+x^{18} \\
& 1.226993 \ldots \quad \Phi_{1}^{2}(x) \Phi_{2}^{2}(x) \Phi_{3}^{2}(x) \Phi_{4}(x) \Phi_{6}(x) \Phi_{12}^{2}(x)+x^{10} \\
& 1.227785 \ldots \quad \Phi_{1}^{2}(x) \Phi_{2}^{2}(x) \Phi_{3}^{2}(x) \Phi_{4}(x) \Phi_{6}(x)+x^{6} \\
& 1.228140 \ldots \quad\left(\Phi_{1}^{2}(x) \Phi_{4}(x) \Phi_{5}(x) \Phi_{12}(x) \Phi_{13}(x) \Phi_{36}(x)-x^{18}\right) / \Phi_{14}(x) \\
& 1.229482 \ldots \quad\left(\Phi_{1}^{2}(x) \Phi_{2}^{2}(x) \Phi_{6}(x) \Phi_{11}(x) \Phi_{13}(x) \Phi_{18}(x) \Phi_{22}(x)+x^{22}\right) / \Phi_{15}(x) \\
& 1.229566 \ldots \quad\left(\Phi_{1}^{2}(x) \Phi_{5}(x) \Phi_{7}(x) \Phi_{36}(x)-x^{12}\right) / \Phi_{6}(x) \\
& 1.229999 \ldots \quad \Phi_{1}^{2}(x) \Phi_{2}^{2}(x) \Phi_{3}(x) \Phi_{6}(x) \Phi_{17}(x) \Phi_{22}(x)+x^{17}
\end{aligned}
$$

The algorithm finds the polynomials with the 37 smallest known measures greater than 1. Table 1 shows how each of the top thirty polynomials (all the known 
polynomials having measure less than 1.23) may be expressed as a slightly perturbed product of cyclotomic polynomials, or a factor of such a product.

Lehmer's polynomial (or this polynomial in $x^{k}$ ) is detected 241 times using this algorithm.

We find 177 polynomials with measure less than 1.3 that do not appear in previous lists and do not seem to be associated with any of the limit points (3.2), (3.3), or (3.4) of Mahler measures. Only 11 of these have degree 30 or 32, and none have degree less than 30. This supports Boyd's heuristic of restricting to polynomials with height 1 in searches of higher degrees [2]. Among these 177 polynomials, the one with the smallest measure is

$$
\begin{aligned}
x^{32}+x^{31}+x^{30}+ & x^{29}-x^{27}-x^{26}-2 x^{25}-x^{24}+x^{21}+x^{20} \\
& -x^{16}+x^{12}+x^{11}-x^{8}-2 x^{7}-x^{6}-x^{5}+x^{3}+x^{2}+x+1 .
\end{aligned}
$$

Its measure is $1.236083 \ldots$, the 47 th smallest Mahler measure greater than 1 known, and the smallest known among primitive, irreducible, noncyclotomic polynomials of degree 32. (We say a polynomial is primitive if it cannot be written as $f\left(x^{k}\right)$ for some $k>1$.) We also note a new record polynomial of degree 34 . Its measure is $1.220287 \ldots$, the 16 th smallest known, but it is associated with the smallest known limit point of Mahler measures.

We find that each of the coefficient adjustments considered is very successful in creating polynomials with small Mahler measure. Through degree 56, the middle coefficient shift (3.5) finds 1037 primitive, irreducible polynomials with measure greater than 1 and less than 1.3. The perturbation (3.6) detects 990 polynomials, and the adjustments (3.7) and (3.8) together find 1031 polynomials.

A Salem number is a real algebraic integer greater than 1, all of whose conjugates lie inside or on the unit circle, with at least one conjugate on the unit circle. The smallest known Salem number is $1.1762808 \ldots$, the real root larger than one of Lehmer's polynomial. Our algorithm finds no new Salem numbers, and detects only 23 of the 43 previously known Salem numbers less than 1.3.

A complete list of the known primitive, irreducible, noncyclotomic polynomials with measure less than 1.3 and degree at most 64 may be found at the first author's World Wide Web site, accessible from the Number Theory Web.

\section{Shifted CyClotomics that Remain CyClotomic}

Suppose $F(x)$ and $G(x)$ are products of cyclotomic polynomials,

$$
F(x)=\prod_{n \geq 1} \Phi_{n}(x)^{e(n)}, \quad G(x)=\prod_{n \geq 1} \Phi_{n}(x)^{f(n)},
$$

with even degree $2 N$,

$$
2 N=\sum_{n \geq 1} e(n) \varphi(n)=\sum_{n \geq 1} f(n) \varphi(n),
$$

and

$$
F(x)-G(x)=l x^{N}
$$

for some positive integer $l$. Then $l \leq 4$ by Theorem 2.1. We show that the polynomials allowing $l=3$ belong to the same family as those allowing $l=4$. 
Theorem 4.1. If $F(x)$ and $G(x)$ are products of cyclotomic polynomials of degree $2 N$ for which (4.2) holds with $l=3$ or 4 , then $F(x)$ and $G(x)$ must arise from a four-shift sequence of the form

$$
\left(x^{N}-1\right)^{2}, x^{2 N}-x^{N}+1, x^{2 N}+1, x^{2 N}+x^{N}+1,\left(x^{N}+1\right)^{2} .
$$

Although we need only consider the case $l=3$, we prove the theorem without this assumption, as the extra work required is minimal and it seems of some interest that the result follows from purely arithmetic considerations.

We require a result of $\mathrm{E}$. T. Lehmer [8] on the resultant of two cyclotomic polynomials.

Lemma 4.2. Suppose $n>m$. Then

$$
\operatorname{Res}\left(\Phi_{n}, \Phi_{m}\right)= \begin{cases}p^{\varphi(m)} & \text { if } n=m p^{k}, p \text { a prime } \\ 1 & \text { otherwise. }\end{cases}
$$

Proof of Theorem 4.1. By Lemma 2.3, we may assume the exponents in (4.1) satisfy

$$
0 \leq e(n) \leq 2, \quad 0 \leq f(n) \leq 2
$$

Suppose that $p$ is a prime with $p^{\alpha(p)} \| l$ for some $\alpha(p) \geq 1$, and that $n$ and $r$ are integers such that $(p, n)=1, e\left(n p^{r}\right) \neq 0$, and $e\left(n p^{j}\right)=f\left(n p^{j}\right)=0$ for all $j>r$ (reversing the roles of $e$ and $f$ if necessary). Taking resultants, we have

$$
\begin{aligned}
l^{\varphi\left(n p^{r}\right)} & =\operatorname{Res}\left(F-l x^{N}, \Phi_{n p^{r}}\right) \\
& =\operatorname{Res}\left(G, \Phi_{n p^{r}}\right) \\
& =\prod_{m \geq 1} \operatorname{Res}\left(\Phi_{m}, \Phi_{n p^{r}}\right)^{f(m)},
\end{aligned}
$$

and comparing powers of $p$,

$$
\alpha(p) \varphi\left(n p^{r}\right)=\sum_{j=0}^{r-1} f\left(n p^{j}\right) \varphi\left(n p^{j}\right) .
$$

Thus $r \geq 1$ and $f\left(n p^{j}\right) \neq 0$ for some $j$ with $0 \leq j<r$. Now $f\left(n p^{j}\right) \leq 2$, so

$$
\alpha(p)(p-1) \leq 2\left(1+(p-1)\left(1+p+\ldots+p^{r-2}\right)\right) / p^{r-1}=2 .
$$

Thus $p=3$ and $\alpha(3)=1$, or $p=2$ and $\alpha(2)=1$ or 2 , and so $l=1,2,3,4$, 6 , or 12. Moreover, if $p=3$ or $p=2$ and $\alpha(2)=2$ we have equality in (4.4), and hence

$$
f(n)=f(p n)=f\left(p^{2} n\right)=\ldots=f\left(p^{r-1} n\right)=2 .
$$

Since $f\left(n p^{j}\right) \neq 0$ for some $j$, we find similarly that

$$
l^{\varphi\left(n p^{j}\right)}=\operatorname{Res}\left(F, \Phi_{n p^{j}}\right)
$$

and so

$$
\alpha(p) \varphi\left(n p^{j}\right)=e\left(n p^{r}\right) \varphi\left(n p^{j}\right)+\sum_{i=0}^{r-1} e\left(n p^{i}\right) \varphi\left(n p^{\min \{i, j\}}\right) .
$$

If $p=3$ or $p=2$ and $\alpha(2)=2$, we have $e\left(n p^{i}\right)=0$ for $0 \leq i<r$ by (4.5), so (4.6) becomes

$$
\alpha(p)=e\left(n p^{r}\right)
$$


and if $p=2$ and $\alpha(2)=1$, then (4.6) shows that $e\left(2^{r} n\right)=1$ and $e\left(2^{i} n\right)=0$ for $0 \leq i<r$.

We now dismiss the cases $l=6$ and $l=12$. Select $m$ to be the largest integer such that $(m, 3)=1$ and either $e(m) \neq 0$ or $f(m) \neq 0$; then select $k$ to be the largest integer such that $e\left(3^{k} m\right) \neq 0$ or $f\left(3^{k} m\right) \neq 0$. Assume $e\left(3^{k} m\right) \neq 0$. As $3 \mid l$, we have $e\left(3^{k} m\right)=1$ and $f(m)=2$. But if $4 \mid l$, we must have $e\left(3^{k} m\right)=2$, and if $2 \| l$, then $e\left(2^{i} m\right) \neq 0$ for some $i \geq 1$, contradicting our choice of $m$.

If $l=4$, all the nonzero $e(n)$ and $f(n)$ are 2 , so we write

$$
F(x)=u^{2}(x), \quad G(x)=v^{2}(x), \quad(u(x)+v(x))(u(x)-v(x))=4 x^{N}
$$

for some polynomials $u(x)$ and $v(x)$ of degree $N$, and so

$$
\begin{gathered}
u(x)+v(x)= \pm 2^{i} x^{N-j} \\
u(x)-v(x)= \pm 2^{2-i} x^{j}
\end{gathered}
$$

for some $0 \leq i \leq 2$ and $0 \leq j \leq N$. Thus

$$
\begin{aligned}
& F(x)=\left(2^{i-1} x^{N-j}+2^{1-i} x^{j}\right)^{2}, \\
& G(x)=\left(2^{i-1} x^{N-j}-2^{1-i} x^{j}\right)^{2} .
\end{aligned}
$$

Since $F(x)$ and $G(x)$ are products of cyclotomic polynomials, we must have $i=1$ and $j=0$ or $N$, and

$$
F(x)=\left(x^{N}+1\right)^{2}, \quad G(x)=\left(x^{N}-1\right)^{2}
$$

as claimed.

If $l=3$, we define the sets

$$
\begin{aligned}
& S=\left\{n: e\left(n 3^{r(n)}\right) \neq 0, e\left(n 3^{j}\right)=f\left(n 3^{j}\right)=0, j>r(n)\right\}, \\
& T=\left\{n: f\left(n 3^{r(n)}\right) \neq 0, e\left(n 3^{j}\right)=f\left(n 3^{j}\right)=0, j>r(n)\right\} .
\end{aligned}
$$

By (4.5) and (4.7) we may write

$$
\begin{aligned}
& F(x)=\prod_{n \in S} \Phi_{n 3^{r(n)}}(x) \prod_{m \in T} \prod_{j=0}^{r(m)-1} \Phi_{m 3^{j}}^{2}(x), \\
& G(x)=\prod_{n \in T} \Phi_{n 3^{r(n)}}(x) \prod_{m \in S} \prod_{j=0}^{r(m)-1} \Phi_{m 3^{j}}^{2}(x) .
\end{aligned}
$$

Observing the identities

$$
\Phi_{n p^{k}}(x)=\frac{\Phi_{n}\left(x^{p^{k}}\right)}{\Phi_{n}\left(x^{p^{k-1}}\right)}, \quad \prod_{j=0}^{k-1} \Phi_{n p^{j}}(x)=\Phi_{n}\left(x^{p^{k-1}}\right)
$$

for $(n, p)=1$, and setting

$$
\begin{aligned}
& u(x)=\prod_{n \in S} \Phi_{n}\left(x^{3^{r(n)-1}}\right) \\
& v(x)=\prod_{n \in T} \Phi_{n}\left(x^{3^{r(n)-1}}\right)
\end{aligned}
$$


we obtain

$$
\begin{aligned}
& F(x)=\frac{u\left(x^{3}\right)}{u(x)} v^{2}(x), \\
& G(x)=\frac{v\left(x^{3}\right)}{v(x)} u^{2}(x) .
\end{aligned}
$$

Thus $\operatorname{deg} u+\operatorname{deg} v=N$,

$$
u\left(x^{3}\right) v^{3}(x)-u^{3}(x) v\left(x^{3}\right)=3 x^{N} u(x) v(x),
$$

and by replacing $u(x)$ and $v(x)$ with $-u(x)$ and $-v(x)$ if necessary, we may assume $u(0)=v(0)=1$. Let $k$ be the smallest integer such that the coefficient of $x^{k}$ in $u(x)$ and $v(x)$ differs, and write

$$
u(x)=p(x)+x^{k} s(x), \quad v(x)=p(x)+x^{k} t(x),
$$

where $p(x)$ has degree at most $k-1$. Substituting into (4.8) yields

$$
3 p\left(x^{3}\right) p^{2}(x)(t(x)-s(x)) x^{k}+w(x) x^{2 k}=3 x^{N} u(x) v(x)
$$

for some polynomial $w(x)$. As $p(0)=u(0)=v(0)=1$ and $s(0) \neq t(0)$, we have $k=N$, and (4.9) shows that one of $u(x)$ and $v(x)$ has degree 0 and the other has degree $N$. Therefore either $u(x)=1$ and $v(x)=1 \pm x^{N}$, or $u(x)=1 \pm x^{N}$ and $v(x)=1$. Thus

$$
\begin{gathered}
F(x)=\left(x^{N}+1\right)^{2}, \quad G(x)=x^{2 N}-x^{N}+1, \\
F(x)=x^{2 N}+x^{N}+1, \quad G(x)=\left(x^{N}-1\right)^{2}
\end{gathered}
$$

are the only solutions.

4.1. Shifts of Length 1 or $\mathbf{2}$. We derive some restrictions on products of cyclotomic polynomials whose roots remain on the unit circle when their middle coefficient is shifted by 2 , and we describe the polynomials found by our algorithm that remain products of cyclotomic polynomials when their middle coefficient is shifted by 1 or 2 .

Suppose $l=2$, and choose $n$ and $r$ so that $n$ is odd, $e\left(n 2^{r}\right) \neq 0$, and $e\left(n 2^{j}\right)=$ $f\left(n 2^{j}\right)=0$ for $j>r$. We have seen that $e\left(n 2^{r}\right)=1, e\left(n 2^{j}\right)=0$ for $j<r$, and (4.3) becomes

$$
2^{r-1}=f(n)+\sum_{j=1}^{r-1} 2^{j-1} f\left(2^{j} n\right) .
$$

The sequence $\left\{f\left(2^{j} n\right)\right\}$ for $0 \leq j \leq r-1$ must therefore take on one of the five forms

$$
\begin{gathered}
2, \ldots, 2,0 \\
0, \ldots, 0,2, \\
1, \ldots, 1 \\
2, \ldots, 2,0,1, \ldots, 1 \\
0, \ldots, 0,2,1, \ldots, 1 .
\end{gathered}
$$


Each of these configurations can occur. For example,

$$
\begin{gathered}
\Phi_{2^{r}}(x)=\Phi_{1}^{2}(x) \Phi_{2}^{2}(x) \cdots \Phi_{2^{r-2}}^{2}(x)+2 x^{2^{r-2}} \\
\Phi_{2^{r-1}}^{2}(x)=\Phi_{2^{r}}(x)+2 x^{2^{r-1}}, \\
\left(\Phi_{p}(x) \Phi_{2 p}(x) \cdots \Phi_{2^{r-1} p}(x)\right)\left(\Phi_{2^{j}}^{2}(x) \Phi_{2^{j+1}}(x) \cdots \Phi_{2^{r-1}}(x)\right)\left(\frac{x^{2^{r-1} p-2^{j-1}}+1}{x^{2^{j-1}}+1}\right) \\
=\Phi_{2^{r} p}(x) \Phi_{2^{r}}(x)\left(\frac{x^{2^{r-1} p-2^{j-1}}-1}{x^{2^{j-1}}-1}\right)+2 x^{2^{r-1} p-2^{j-1}} \\
\left(\Phi_{p}(x) \cdots \Phi_{2^{r-1} p}(x)\right)\left(\Phi_{1}^{2}(x) \cdots \Phi_{2^{j-1}}^{2}(x) \Phi_{2^{j+1}}(x) \cdots \Phi_{2^{r-1}}(x)\right)\left(\frac{x^{2^{r-1} p-2^{j-1}}-1}{x^{2^{j-1}}-1}\right) \\
=\Phi_{2^{r} p}(x) \Phi_{2^{r}}(x)\left(\frac{x^{2^{r-1} p-2^{j-1}}+1}{x^{2^{j-1}}+1}\right)-2 x^{2^{r-1} p-2^{j-1}} .
\end{gathered}
$$

Our search finds many products of cyclotomic polynomials that remain products of cyclotomic polynomials after shifting their middle coefficient by 2 . All of the primitive examples found have the form

$$
\frac{\left(x^{N+1}+1\right)\left(x^{N}-1\right)}{x-1}+2 x^{N}=\frac{\left(x^{N+1}-1\right)\left(x^{N}+1\right)}{x-1}
$$

with $N$ a positive integer, or

$$
\frac{\left(x^{N+2}+1\right)\left(x^{N}-1\right)}{x^{2}-1}+2 x^{N}=\frac{\left(x^{N+2}-1\right)\left(x^{N}+1\right)}{x^{2}-1}
$$

with $N$ odd.

Our search also finds many products of cyclotomic polynomials that allow a shift of exactly 1 . Taking the symmetry of $f(x)$ and $f(-x)$ into account, we detect 449 essentially different primitive pairs of such polynomials through degree 64 . Of these, 401 have the form

$$
\frac{\left(x^{N+m}+s\right)\left(x^{N+n}+r\right)}{\left(x^{m}-1\right)\left(x^{n}-r s\right)}+s x^{N}=\frac{\left(x^{N+m+n}+r\right)\left(x^{N}+s\right)}{\left(x^{m}-1\right)\left(x^{n}-r s\right)},
$$

where $r$ and $s$ are \pm 1 and either $r=s=-1$ or $r=-s$, and $N, m$, and $n$ satisfy certain divisibility conditions so that the two rational functions are in fact polynomials. All but 9 of the remaining pairs are described by one of the families

$$
\begin{gathered}
\frac{\left(x^{N}-s\right)\left(x^{N+2}-s\right)}{x^{2}+s x+1}+s x^{N}=\frac{x^{2 N+2}+x^{N+1}+1}{x^{2}+s x+1}, \\
\frac{\left(x^{N}+r\right)\left(x^{N+1}+s\right)\left(x^{2 N+1}+r s\right)}{x^{2}-r s x+1}+x^{2 N}=\frac{\left(x^{2 N}+r x^{N}+1\right)\left(x^{2 N+2}+s x^{N+1}+1\right)}{x^{2}-r s x+1} \\
\frac{\left(x^{N}+s\right)\left(x^{N+2}-s\right)\left(x^{2 N+2}-1\right)}{x^{4}+x^{2}+1}+x^{2 N}=\frac{\left(x^{2 N}+s x^{N}+1\right)\left(x^{2 N+4}-s x^{N+2}+1\right)}{x^{4}+x^{2}+1}
\end{gathered}
$$


where $r$ and $s$ are \pm 1 and $N$ satisfies certain constraints modulo 6 . The 9 exceptional pairs are listed below:

$$
\begin{gathered}
\Phi_{4}(x) \Phi_{30}(x)+x^{5}=\Phi_{3}(x) \Phi_{24}(x), \\
\Phi_{4}(x) \Phi_{16}(x) \Phi_{30}(x)+x^{9}=\Phi_{5}(x) \Phi_{18}(x) \Phi_{24}(x), \\
\Phi_{2}^{2}(x) \Phi_{6}^{2}(x) \Phi_{12}(x) \Phi_{14}(x) \Phi_{30}(x)+x^{12}=\Phi_{5}(x) \Phi_{20}(x) \Phi_{21}(x), \\
\Phi_{1}^{2}(x) \Phi_{2}^{2}(x) \Phi_{3}(x) \Phi_{4}(x) \Phi_{5}(x) \Phi_{26}(x)+x^{12}=\Phi_{30}(x) \Phi_{60}(x), \\
\Phi_{3}(x) \Phi_{24}(x) \Phi_{50}(x)+x^{15}=\Phi_{4}(x) \Phi_{16}(x) \Phi_{30}(x) \Phi_{36}(x), \\
\Phi_{1}^{2}(x) \Phi_{2}^{2}(x) \Phi_{3}(x) \Phi_{4}(x) \Phi_{5}(x) \Phi_{6}^{2}(x) \Phi_{8}(x) \Phi_{24}(x) \Phi_{42}(x)+x^{20}=\Phi_{60}(x) \Phi_{70}(x), \\
\Phi_{3}(x) \Phi_{24}(x) \Phi_{27}(x) \Phi_{50}(x)+x^{24}=\Phi_{4}(x) \Phi_{5}(x) \Phi_{18}(x) \Phi_{20}(x) \Phi_{36}(x) \Phi_{40}(x), \\
\Phi_{1}^{2}(x) \Phi_{2}^{2}(x) \Phi_{3}(x) \Phi_{4}(x) \Phi_{6}(x) \Phi_{7}(x) \Phi_{8}(x) \Phi_{24}(x) \Phi_{50}(x)+x^{24}=\Phi_{70}(x) \Phi_{84}(x), \\
\Phi_{3}(x) \Phi_{6}(x) \Phi_{27}(x) \Phi_{30}(x) \Phi_{56}(x)+x^{27}=\Phi_{4}(x) \Phi_{5}(x) \Phi_{20}(x) \Phi_{40}(x) \Phi_{45}(x) .
\end{gathered}
$$

\section{REFERENCES}

[1] F. Amoroso, Algebraic numbers close to 1 and variants of Mahler's measure, J. Number Theory 60 (1996), 80-96. MR 97g:11075

[2] D. W. Boyd, Reciprocal polynomials having small measure. I, II, Math. Comp. 35 (1980), 1361-1377; II 53 (1989), 355-357, S1-S5. MR 82a:30005; MR 89m:30013

[3] _ Speculations concerning the range of Mahler's measure, Canad. Math. Bull. 24 (1981), 453-469. MR 83h:12002

[4] D. W. Boyd and H. L. Montgomery, Cyclotomic partitions, in Number Theory (R. A. Mollin, ed.), Walter de Gruyter, 1990, 7-25. MR 92b:11073

[5] E. Dobrowolski, On a question of Lehmer and the number of irreducible factors of a polynomial, Acta Arith. 34 (1979), 391-401. MR 80i:10040

[6] E. Hille, Analytic Function Theory, Vol. II, Chelsea, 1987.

[7] D. H. Lehmer, Factorization of certain cyclotomic functions, Ann. of Math. (2) 34 (1933), 461-479.

[8] E. T. Lehmer, A numerical function applied to cyclotomy, Bull. Amer. Math. Soc. 36 (1930), 291-298.

[9] M. J. Mossinghoff, Algorithms for the determination of polynomials with small Mahler measure, Ph.D. Thesis, The University of Texas at Austin, 1995.

[10] M. J. Mossinghoff, Polynomials with small Mahler measure, Math. Comp. 67 (1998), 16971705.

[11] G. Pólya and G. Szegö, Problems and Theorems in Analysis, II, Springer-Verlag, 1976. MR 57:5529

[12] A. G. Postnikov, Introduction to Analytic Number Theory, Transl. Math. Monogr., vol. 68, Amer. Math. Soc., 1988. MR 89a:11001

[13] U. Rausch, On a theorem of Dobrowolski about conjugate numbers, Colloq. Math. 50 (1985), 137-142. MR 87i:11144

[14] J. H. Silverman, Exceptional units and numbers of small Mahler measure, Experiment. Math. 4 (1995), 69-83. MR 96j:11150

[15] C. J. Smyth, On the product of the conjugates outside the unit circle of an algebraic integer, Bull. London Math. Soc. 3 (1971), 169-175. MR 44:6641

[16] J. Stoer and R. Bulirsch, Introduction to Numerical Analysis, Springer-Verlag, 1980. MR 83d:65002 
Department of Mathematics, University of Texas at Austin, Austin, Texas 78712

Current address: Department of Mathematical Sciences, Appalachian State University, Boone, North Carolina 28608

E-mail address: mjm@math.appstate.edu

Department of Mathematics, University of Texas at Austin, Austin, Texas 78712

Current address: Department of Mathematics and Statistics, University of Ottawa, 585 King Edward Ave., Ontario K1N 6N5, Canada

E-mail address: pinner@mathstat.uottawa.ca

Department of Mathematics, University of Texas at Austin, Austin, Texas 78712

E-mail address: vaaler@math.utexas.edu 